\title{
Ambulatory antibiotic prescribing for acute bronchitis and cough and hospital admissions for respiratory infections: time trends analysis
}

\author{
Arch G Mainous III $^{1} \quad$ Sonia Saxena ${ }^{2} \quad$ William J Hueston ${ }^{1} \quad$ Charles J Everett ${ }^{1} \quad$ Azeem Majeed²
}

J R Soc Med 2006;99:358-362

\section{SUMMARY}

Objectives To examine the relationship between ambulatory antibiotic prescribing for acute bronchitis and cough with hospital admissions for respiratory infections in the USA between 1996 and 2003.

Design Analysis of data on antibiotic prescribing for episodes of acute bronchitis/cough illness in ambulatory care and hospitalization for respiratory infections for adults between 1996 and 2003 in the USA.

Setting USA: ambulatory prescribing behaviour was derived from the National Ambulatory Medical Care Survey and the National Hospital Ambulatory Medical Care Survey while hospitalizations in acute care hospitals were assessed in the National Hospital Discharge Survey.

Participants Adults 18-64 years old.

Interventions None.

Main outcome measures Proportion of visits for acute bronchitis/cough receiving a prescription for antibiotics and hospitalization for respiratory infections.

Results Ambulatory antibiotic prescribing practices for acute bronchitis/cough and hospitalizations for respiratory infections exhibited non-linear patterns over the 8 year period. However, antibiotic prescribing practices for acute bronchitis/cough and hospitalizations for respiratory infections had a weak/moderate negative association. For three of the seven yearly changes in prescribing and hospitalizations as one increased the other decreased $(P<0.01)$.

Conclusions Ambulatory antibiotic prescribing for respiratory tract infections was inversely associated with hospital admissions for respiratory tract infections.

\section{INTRODUCTION}

In recent years, antibiotic resistance has increased worldwide. The amount of antibiotics consumed in a community

'Department of Family Medicine, Medical University of South Carolina, Charleston, South Carolina, USA; ${ }^{2}$ Department of Primary Care and Social Medicine, Imperial College, London, UK

Correspondence to: Arch G. Mainous III PhD is directly related to the amount of antibiotic resistance found in the community. ${ }^{1,2}$ Inappropriate use of antibiotics, particularly for respiratory infections, has contributed to the major public health problem of antibiotic resistance. Domestic interventions in the USA have decreased inappropriate antibiotic prescribing in general and in particular for respiratory infections. ${ }^{3,4}$

Prescribing of antibiotics for acute bronchitis has been the focus of guidelines designed to decrease prescribing for this condition and thereby decrease antibiotic resistance. ${ }^{5,6}$ The guideline endorsed by the Centers for Disease Control and Prevention, the American Academy of Family Physicians, the American College of Physicians, American Society or Internal Medicine, and the Infectious Diseases Society of America recommended that antibiotic treatment of uncomplicated acute bronchitis should not be given routinely, regardless of duration of cough. However, the Cochrane review of the trials of treatment for acute bronchitis as well as several other meta-analyses have indicated that antibiotics have a moderate beneficial effect in the treatment of acute bronchitis. ${ }^{7-9}$ However, currently we have not been able to reliably identify the subgroups of patients that would benefit from antibiotics. Perhaps, more importantly, the guideline for acute bronchitis indicates that the evaluation of adults with an acute cough illness or presumptive acute bronchitis must first focus on ruling out serious illness like pneumonia. 5,6 Thus, it is unclear whether an unintended consequence of a strategy to decrease antibiotic resistance has been to decrease the use of antibiotics in cases where antibiotics might be beneficial.

Data from the UK provide mixed results on whether decreased antibiotic prescribing has increased hospitalizations or complications. One study suggested that while antibiotic prescribing in the population for all conditions had decreased, hospitalizations for respiratory infections had increased. ${ }^{10}$ Another study indicated that, while antibiotic prescribing for all conditions had decreased, there was no significant increase in suppurative complications like peritonsillar abscess, mastoiditis or rheumatic fever in children. ${ }^{11}$ A third study examining data for the year $1997-$ 1998 suggested that higher use of penicillin in the community was associated with significantly fewer 
admissions for major complications of respiratory tract infections. ${ }^{12}$ None of these studies directly linked antibiotic prescribing behaviour for treatment for specific conditions (e.g. bronchitis).

In the USA, although antibiotic prescribing for acute bronchitis among adults decreased during the 1990s, it is unclear whether changes in antibiotic prescribing were associated with hospitalizations for respiratory illness. The purpose of this study was therefore to examine the relationship between ambulatory physician antibiotic prescribing behaviour for acute bronchitis and cough with hospital admissions for respiratory infections in the USA between 1998 and 2003.

\section{METHODS}

The current study is focused on adults aged 18-64 years. Data were obtained from three annual nationally representative surveys of the USA collected from 19962003. We focused on individuals 18-64 because they account for more than $66 \%$ of ambulatory visits for acute bronchitis/cough during the 8-year period, and older adults have more co-morbidities affecting likelihood of hospitalization for respiratory infections regardless of the use of antibiotics. Two of the surveys focus on provider behaviour in ambulatory care. The National Ambulatory Medical Care Survey (NAMCS) and the National Hospital Ambulatory Medical Care Survey (NHAMCS) focus on provider behaviour in ambulatory care. The National Hospital Discharge Survey (NHDS) focuses on hospitalizations in acute care hospitals.

\section{Ambulatory prescribing behaviour for acute bronchitis/cough}

The NAMCS samples non-federally employed office-based physicians who are primarily engaged in direct patient care; NHAMCS collects data from hospital outpatient and emergency departments in the USA and excludes federal, military, and Veterans Affairs hospitals. These two surveys are often combined by the National Center for Health Statistics (NCHS) to provide a nationally representative picture of ambulatory care. ${ }^{13}$ The surveys have a threetiered multistage probability design based on geographic location, physician specialty and individual visits within the practice. The NCHS weighs each visit by taking into account practice location and physician specialty. The weighting calculations also account for practices that were invited to participate but decline to do so. This weighting of survey clusters allows for data extrapolation to national estimates for all survey items.

For each patient visit up to three diagnoses (one primary and two secondary) are coded according to the International Classification of Diseases, 9th revision, Clinical Modification
(ICD-9-CM).The NAMCS and NHAMCS drug lists use generic drug names. For the current study we focused on prescribing for acute bronchitis (466), bronchitis not otherwise specified (490) and cough (786.2) as the primary diagnosis. Up to six medications per visit can be recorded at the visit. Medications given either as samples or by prescription are included, and are referred to as prescribed in this study. Antibiotic prescription for each visit was identified by using both trade and generic named antimicrobial agents assigned by NAMCS and NHAMCS to each medication prescribed. Because the diagnosis cannot be directly linked to the medication, and to minimize the likelihood that an antibiotic prescription was unrelated to the principal diagnosis, we counted only those prescriptions entered as the primary medication related to the office visit on the patient record form.

\section{Hospitalizations for respiratory tract infections}

NHDS is conducted each year by the NCHS to collect information regarding the characteristics of discharged inpatients. Approximately 500 short-term, non-federal hospitals across the country are used in the sample. From these hospitals, nearly 270000 inpatient records are collected each year.

As with the NAMCS and NHAMCS, the NHDS does not track personal medical care, but rather collects data on individual inpatient stays. These data provide a summary view of the burden of illness based on the morbidity of hospitalization. We operationalized inpatient stays for respiratory infections as hospitalizations with an associated ICD-9-CM code for any of the seven following recorded diagnoses. These included:

- pneumonia (481.xx-486.xx)

- acute bronchitis or bronchiolitis (466.xx)

- bronchiectasis (494)

- empyema (510.xx)

- emphysema (492.xx)

- chronic bronchitis (491.xx)

- abscess of lung and mediastinum (513.xx)

- acute respiratory failure (518.81)

- other pulmonary insufficiency, not otherwise classified (518.82).

\section{Analysis}

The NAMCS, NHAMCS and NHDS each are based on a complex survey design that allows the user to make population estimates. Our analysis used the appropriate sampling weights and the analysis was conducted using SUDAAN to account for the complex sampling designs (SUDAAN, Research Triangle Park, NC, USA). 
Table 1 Unwezighted and weighted numbers of ambulatory visits for acute bronchitis/cough illness receiving antibiotic prescriptions, total visits for acute bronchitis/ cough illness, hospitalizations for respiratory infections and total hospitalizations by year in the USA $1996-2003$

\begin{tabular}{lllll}
\hline & $\begin{array}{l}\text { Visits } \\
\text { receiving } \\
\text { antibiotics }\end{array}$ & Total visits & $\begin{array}{l}\text { Hospitalizations } \\
\text { for respiratory } \\
\text { infections }\end{array}$ & $\begin{array}{l}\text { Total } \\
\text { hospitalizations }\end{array}$ \\
\hline 1996 & $3930422(372)^{\star}$ & $5687503(548)$ & $1008406(7886)$ & $16001297(130900)$ \\
1997 & $3957271(344)$ & $6440887(537)$ & $964585(8262)$ & $15811689(137639)$ \\
1998 & $6652658(416)$ & $9205220(630)$ & $1065107(9036)$ & $16458692(141907)$ \\
1999 & $4016105(375)$ & $6999392(631)$ & $1081926(9134)$ & $16399619(139422)$ \\
2000 & $3742685(337)$ & $6632564(609)$ & $1060420(9360)$ & $16397409(146780)$ \\
2001 & $3877099(418)$ & $7450612(684)$ & $1101989(9895)$ & $16974997(154688)$ \\
2002 & $5863047(503)$ & $9384514(810)$ & $1140636(10108)$ & $17837105(156032)$ \\
2003 & $6428012(525)$ & $9892260(804)$ & $1249629(10160)$ & $18339518(151716)$
\end{tabular}

*Weighted number with unweighted number in parentheses

Since each year was a separate national estimate, we analysed the prescribing behaviour estimates from each year controlling for the design effect in the NAMCS/NHAMCS. We computed $\chi^{2}$ statistics based on the weighted estimates using Epi-Info (Centers for Disease Control and Prevention, Atlanta, GA, USA). In an effort to examine the relationship between antibiotic prescribing and hospitalizations, we computed Spearman correlation coefficients on the weighted population estimates.

\section{RESULTS}

A large number of visits were for acute bronchitis or cough in each of the investigated years. Although the analysis is based on weighted national estimates, it is useful to note the number of unweighted events used to make the national estimates. The unweighted number of visits with the most general classification of acute bronchitis/cough illness in the NAMCS/NHAMCS ranged from 537-810 for the 8 years analysed in this study, corresponding to a weighted estimate of visits ranging from 5687503 to 9892260 (Table 1). The total unweighted number of hospitalizations included in the NHDS ranged from 130900 to 156032 for the 8 years, corresponding to a weighted national estimate of hospitalizations ranging from 15811689 to 18339518 across the years.

Figure 1 indicates that ambulatory antibiotic prescribing for acute bronchitis or cough dropped in the late 1990s and increased in the new century. A nonlinear relationship was yielded for both antibiotic prescribing and hospitalizations for respiratory infections with a negative correlation between them (Spearman's $r=-0.22$ ). In three of the seven change periods investigated, as prescribing of antibiotics for acute bronchitis/cough increased or decreased the proportion of total hospitalizations did the
We also examined the types of respiratory conditions that resulted in hospital admissions over time (Table 3). The proportion of hospitalizations for pneumonia, chronic bronchitis/emphysema, or other respiratory infections of the total respiratory hospitalizations for respiratory infections remained relatively stable throughout the 8-year period.

\section{DISCUSSION}

The present study suggests that the behaviour of clinicians regarding prescribing of antibiotics for acute bronchitis/ cough illness in the USA, during the late 1990s and first years of the 21 st century, was associated with hospitalizations for respiratory infections. Although, in general, the relationship is suggestive and not causal our study suggests that as clinicians' decreased antibiotic prescriptions for

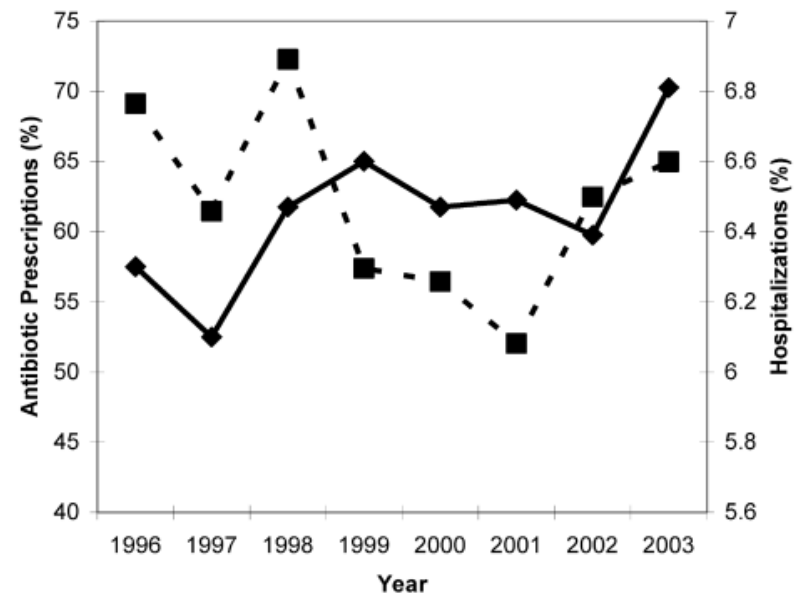

Figure 1 Ambulatory visits for acute bronchitis/cough illness receiving antibiotics as a percentage of all visits for acute bronchitis/cough illness and hospitalizations for respiratory infections as a percentage of all hospitalizations. Antibiotics -7; hospitalizations - 
Table 2 Percentage change from year to year in ambulatory visits for acute bronchitis/cough illness that received antibiotic prescriptions as a percentage of all visits, and in hospitalizations for respiratory infections as a percentage of all hospitalizations

\begin{tabular}{lll}
\hline & $\begin{array}{l}\text { Antibiotic } \\
\text { prescriptions } \\
\text { (\% change) }\end{array}$ & $\begin{array}{l}\text { Hospitalizations } \\
\text { (\% change) }\end{array}$ \\
\hline $1996-1997$ & $-11^{*}$ & $-3.2^{*}$ \\
$1997-1998$ & $+18^{*}$ & $+6.1^{*}$ \\
$1998-1999$ & $-21^{*}$ & $+2.0^{*}$ \\
$1999-2000$ & $-2^{*}$ & $-2.0^{*}$ \\
$2000-2001$ & $-8^{*}$ & $+0.3^{*}$ \\
$2001-2002$ & $+20^{*}$ & $-1.5^{\star}$ \\
$2002-2003$ & $+4^{*}$ & $+6.6^{\star}$ \\
\hline
\end{tabular}

*Significant at $P<0.01$

acute bronchitis/cough illness fell, hospitalizations for respiratory conditions rose.

However, this effect seems to be driven by variations in fewer than half of the years investigated. The effect is primarily centred between 1998 and 2002. If the analysis were limited to the years 1998-2002 rather than 19962003, the correlation would be -0.46 rather than the -0.22 that was found for the longer time period. It is important, therefore, to further examine this relationship over time because of the potential for transient changes in the overall relationship.

There are several possibilities that could explain our findings. Because these results are based on overall population effects, there is a good chance that, whilst these two phenomenon are linked together in time, they are not related. Antibiotic prescribing for acute bronchitis and the increase in hospitalizations for respiratory conditions easily could be two independent events.

Clearly, these results alone nor the previous ecological study in the UK can establish how ambulatory antibiotic prescribing and hospitalizations for respiratory infections are related or even whether they are related. ${ }^{10}$ Continued observation of the trends in antibiotic use and hospitalizations for respiratory illnesses may be useful; but in these ecological studies, the people receiving antibiotics may bear no relationship to those who are admitted with respiratory infections. A large observational study of patients with acute bronchitis that can look at individualized risks for hospitalization after antibiotic use and which can screen for the development of colonization with antibiotic resistant organisms would be essential to draw any firmer conclusions.

There are several limitations to this study. First, due to its sampling design and weights, the NAMCS/NHAMCS data presented here theoretically offers accurate estimates of physician behaviour. Although the current analysis uses existing data and the participating physicians were unaware at the time of data collection of the present study's aims, NAMCS/NHAMCS data are based on physician self-reports and may be affected by participants' preference to be seen as practising in a socially desirable fashion. The actual practice of participants in these surveys could be different than what they indicated on their data collection instruments or may differ in the times when they are not participating as subjects.

Second, this study links data regarding estimates of antibiotic prescribing within ambulatory care representative of the entire USA with estimates of hospitalizations for the entire population. These types of data are very useful and are available in the USA but are uncommon in many European countries. However, the data allow for an ecological study but do not allow us to investigate for a cohort of individuals what happens in the course of their illness when they receive or do not receive antibiotics. The data do provide a general picture of the relationship of prescribing behaviour to morbidity.

It is possible that physicians sometimes misdiagnose early pneumonia as acute bronchitis because there is a great deal of overlap in the two syndromes. When antibiotics are used routinely for acute bronchitis, this error never has any clinical consequences. But once clinicians begin to restrict antibiotic use for acute bronchitis, those cases that were misclassified may advance into more serious illnesses. This relationship would imply that physicians may need to be more cautious and assure that they rule out other more serious lower respiratory infections before making the diagnosis of acute bronchitis.

In conclusion, our findings suggest that reduced ambulatory physician antibiotic prescribing behaviour for respiratory tract infections was associated with higher hospital admissions for respiratory tract infections. Whether

Table 3 Hospitalizations for selected respiratory infections as a percentage of all hospitalizations

\begin{tabular}{llll}
\hline Year & Pneumonia & $\begin{array}{l}\text { Chronic } \\
\text { bronchitis } \\
\text { and emphysema }\end{array}$ & $\begin{array}{l}\text { Other } \\
\text { respiratory } \\
\text { infections }\end{array}$ \\
\hline 1996 & 3.25 & 1.75 & 1.30 \\
1997 & 3.15 & 1.77 & 1.18 \\
1998 & 3.38 & 1.84 & 1.25 \\
1999 & 3.36 & 1.86 & 1.37 \\
2000 & 3.33 & 1.84 & 1.30 \\
2001 & 3.53 & 1.71 & 1.25 \\
2002 & 3.27 & 1.82 & 1.30 \\
2003 & 3.69 & 1.84 & 1.28 \\
\hline
\end{tabular}


there is a true negative association between antibiotic prescribing and hospital admissions is an important public health issue and needs to be examined further in both ecological and individual level analyses.

Competing interests None declared.

Sponsorship The project was supported in part by grant 1D12HP00023 from the Health Resources and Services Administration. The authors' work on this manuscript was independent of the funders.

\section{REFERENCES}

1 Austin DJ, Kristinsson KG, Anderson RM. The relationship between the volume of antimicrobial consumption in human communities and the frequency of resistance. Proc Natl Acad Sci USA 1999;96:1152-6

2 Albrich WC, Monnet DL, Harbarth S. Antibiotic selection pressure and resistance in Streptococcus pneumoniae and Streptococcus pyogenes. Emerg Infect Dis 2004;10:514-17

3 Finkelstein JA, Stille C, Nordin J, et al. Reduction in antibiotic use among US children, 1996-2000. Pediatrics 2003;112:620-7

4 Interagency Task Force on Antimicrobial Resistance. A Public Health Action Plan to Combat Antimicrobial Resistance: Part 1: Domestic Issues. Washington, DC: Department of Health and Human Services, 2004
5 Snow V, Mottur-Pilson C, Gonzales R. Principles of appropriate antibiotic use for treatment of acute bronchitis in adults. Ann Intern Med 2001; 134:518-20

6 Gonzales R, Bartlett JG, Besser RE, et al. Principles of appropriate antibiotic use for treatment of uncomplicated acute bronchitis: background. Ann Intern Med 2001;134:521-9

7 Smucny J, Fahey T, Becker L, Glazier R. Antibiotics for acute bronchitis. Cochrane Database Syst Rev 2000; (4): CD000245 [update 2004; (4), CD000245]

8 Smucny JJ, Becker LA, Glazier RH, McIsaac W. Are antibiotics effective treatment for acute bronchitis? A meta-analysis. J Fam Pract 1998;47:453-60

9 Bent S, Saint S, Vittinghoff E, Grady D. Antibiotics in acute bronchitis: a meta-analysis. Am J Med 1999;107:62-7

10 Majeed A, Williams S, Jarman B, Aylin P. Prescribing of antibiotics and admissions for respiratory tract infections in England. BMJ 2004;329:879

11 Sharland M, Kendall H, Yeates D, Randall A, Hughes G, Glasziou P, et al. Antibiotic prescribing in general practice and hospital admissions for peritonsillar abscess, mastoiditis, and rheumatic fever in children: time trend analysis. BMJ 2005;331:328-9

12 Little P, Watson L, Morgan S, Williamson I. Antibiotic prescribing and admissions with major suppurative complications of respiratory tract infections: a data linkage study. Br J Gen Pract 2002;52:187-90

13 Burt CW, Schappert SM. Ambulatory care visits to physician offices, hospital outpatient departments, and emergency departments; United States, 1999-2000. National Center for Health Statistics. Vital Health Stat 2004;13:157 